\title{
Machine Learning Models for Predicting, Understanding and Influencing Health Perception
}

\author{
Ada Aka* \\ Departments of Marketing and Psychology \\ University of Pennsylvania \\ 3720 Walnut St. \\ Philadelphia, PA, 19104 \\ adaaka@wharton.upenn.edu
}

\author{
Sudeep Bhatia \\ Departments of Psychology and Marketing \\ University of Pennsylvania \\ 3720 Walnut St. \\ Philadelphia, PA, 19104 \\ bhatiasu@psych.upenn.edu
}

\section{Author Note}

Funding was received from the National Science Foundation grant SES-1847794, the Alfred P. Sloan Foundation, and Wharton Risk Management Center's Russell Ackoff Doctoral Student Fellowship. We thank Liat Gradstein for excellent research assistantship, and Russell Richie and Wanling Zou for helpful discussion. Correspondence concerning this manuscript should be addressed to Ada Aka (adaaka@wharton.upenn.edu). Our data and models will be accessible at https://osf.io/peym7/. We also thank participants of 2020 Society for Judgment and Decision making for their helpful discussion during the conference presentation. No potential conflict of interest was reported by the authors. 
Machine Learning Models for Predicting, Understanding and Influencing Health Perception 


\begin{abstract}
Lay perceptions of medical conditions and treatments determine people's health behaviors, guide biomedical research funding, and have important consequences for both individual and societal wellbeing. Yet it has been nearly impossible to quantitatively predict lay health perceptions for hundreds of everyday diseases due to the myriad psychological forces governing health-related attitudes and beliefs. Here we present a data-driven approach that uses text explanations on healthcare websites, combined with large-scale survey data, to train a machine learning model capable of predicting lay health perception. We use our model to analyze how language influences health perceptions, interpret the psychological underpinnings of health judgment, and quantify differences between different descriptions of disease states. Our model is accurate, cost-effective, and scalable, and offers researchers and practitioners a new tool for studying health-related attitudes and beliefs.
\end{abstract}


COVID-19 has disrupted millions of lives. Medical authorities have emphasized the importance of face masks as precautionary measures for reducing the spread of the disease, however many people reject this advice, aggravating a major health crisis and endangering the lives of others. Although there are many determinants of face mask-avoidance and related behaviors, one key factor is the perception of the severity of COVID-19 (Cheung, 2020), and researchers have emphasized that understanding such health perceptions is necessary for influencing and improving behavior during the crisis (Bavel et al 2020).

Of course, the importance of health perception in preventative health behavior extends beyond just COVID-19. Significant previous work has shown that the perceived severity of a health problem and the perceived vulnerability of the decision maker are significant factors determining precautionary behavior, in domains as diverse as vaccination and mothers' adherence to a diet prescribed for their obese children (Janz and Becker 1984; Van Der Pligt 1998; Weinstein 2000; Becker et al. 1977). More generally, there is a close relationship between perceptions of health outcomes (e.g., Covid-19 or lung cancer), preventative health decisions (e.g., wearing a mask or exercising regularly), and the likelihood of engaging in risky behaviors (e.g., participating in large group gatherings or smoking) (Brewer et al. 2007; Harrison, Mullen, and Green 1992; Sheeran, Harris, and Epton 2014). Thus, in order to facilitate better decision making and preventative behavior, it is essential to predict and understand how people perceive different diseases and other relevant health-related outcomes (Betsch, Bohm, and Chapman 2015; Li and Chapman 2013; Chapman 2019).

Health perception also has important implications for healthcare funding. Every year, pharmaceutical companies, policy makers, and private foundations allocate large quantities of funds and efforts to improve the outcomes of medical conditions and diseases (e.g., World Health Report 1999). Unsurprisingly, such funding decisions depend on how consumers, voters, and donors perceive the severity of health states, and changes to media coverage and popular perceptions of a disease can have significant effects on how much funding is allocated to health programs aimed at combating the disease (Corbett and Mori 1999; Hudacek et al. 2011). The inclusion of lay people perceptions in health services research can also be quite beneficial, as patients and other stake holders often have insights that complement those of health care professionals (Entwistle et al. 1998; Cornwall and Jewkes 1995). 
How can we predict, understand, and influence people's health perceptions for common disease states? One possibility is to use quantitative measures that evaluate the quality of life implications and assess the severity of different medical conditions associated with the disease state (e.g., Calvert and Freemantle 2003). One of the simplest such measures is the count of raw mortality, i.e. the number of deaths caused by the health condition. A related measure is called the "Years of Life Lost," which weighs the severity of death against the age a person dies. Other measures such as the "disability adjusted life years" (DALYs) and "quality-adjusted life years" (QALYs) account for the quality of life lost due to health conditions that are not fatal.

These measures attempt to help describe the global burdens of diseases and better allocate limited resources (Arnesen and Kapiriri 2004). However, considerable research in psychology, marketing, and policy research has found that such measures do not correlate with people's health state perceptions (Slovic and Peters 2006). That is, people are not actually good at evaluating the objective risk and the severity of different health states or treatments (Lloyd 2001). Rather, their judgments rely on emotion, memory, linguistic, social, and other psychological cues, which occasionally lead to perceptions that deviate from objective measures such as the mortality rates or DALYs (Chapman and Coups 2006; Chapman and Elstein 2000; Peters and Meilleur 2016; Slovic et al 2002). These psychological factors cause variables such as perceived pain, disability, physical distress, anxiety, depression, negative mood, functional inability, permanent state of ill health, and death to be particularly strong predictors of (low) health perceptions (Idler 1993; Kaplan and Camacho 1983; Fýlkesnes and Førde 2002; Farmer and Ferraro 1997; Garrity, Somes, and Marx 1978; Krause and Jay 1994; Hudson and Leventhal 1999).

The best way to predict health perception may thus be to understand the associations between a disease state and variables like perceived pain and disability, and the emotions and cognitions that these variables provoke. These associations depend critically on how information about the health state is communicated (Rothman and Salovey 1997; Keller and Lehmann 2008; Vahabi 2007). A survey conducted by Covello and Peters (2002) revealed that $81 \%$ of the women who participated in a research study on risk perceptions indicated general media (e.g., newspapers, TV, magazines) as their main source of information. More recently, the Internet has not only shaped how people obtain information in many different domains but it also became an important health information resource for millions of people (Atkinson, Saperstein, and Pleis 
2009). In fact, it has been argued that the Internet forms the first opinion and doctor's advice is relegated to the second opinion for the consumers of online health information (Gualtieri 2009b). In a closely related area of research, Reyna highlights the importance of qualitative meaning (i.e., gist) on the interpretations of information and events surrounding risks obtained mainly through text, such as online descriptions (Reyna 2020a, 2020b).

The internet is not only a convenient tool for consumers of health information, it also offers researcher easy access to the information sources at play in health perceptions, and thus can be used by researchers to predict, understand, and even influence them. Our goal, in this paper, is to use information communicated on the internet to model health perceptions for hundreds of common disease states. For this purpose, we use textual information presented on the National Health Service (NHS) website, which is one of the main online sources of health information in the United Kingdom (Powell, Inglis, Ronnie, and Large 2011). We additionally rely on recent advances in machine learning, known as word and sentence embeddings, which can quantify textual content for use in quantitative analysis (Bhatia 2019; Günther, Rinaldi, and Marelli 2019; Devlin 2018; Rogers, Kovaleva, and Rumshisky 2020). Such methods have been shown to achieve state of the art results across different areas of natural language processing, including sentiment analysis and question answering. By using embedding methods to quantify the informational content of health descriptions on the NHS we can build a machine learning model that is capable of predicting health perception given a textual description of a health state.

In Study 1, we illustrate the feasibility of this approach in large-scale study involving participant judgments of 777 distinct disease states. Here we use participant data and text explanations to train our model, and evaluate its predictions on held-out data (that is text explanations and participant evaluations on which the model has not been trained). We also compare the performance of our embedding model with other competing models, including those that rely only on objective statistics like mortality or simpler features extracted from the text data (e.g. text length, concreteness, and sentiment). In Study 2, we use our trained embedding models to interpret how information contained in online text explanations influences perceptions of the severity of health states. In particular, we examine, psychological factors and variables identified in prior research on health perception and use a variety of techniques to observe how strongly our trained models rely on these variables. Finally, in Study 3, we use our embedding models to predict how different descriptions of the same disease state can be associated with different 
perceptions. This study tests the utility of our approach for identifying psychological cues (such as linguistic patterns) that can most accurately communicate risks. If successful, the results of this study could be used to inform policy-insights and behavioral interventions for better health communication.

\section{Study 1: Predicting Health Judgments}

In Study 1, we evaluated the accuracy of our approach for predicting lay people's health perceptions. In particular, we used sentence and word embeddings to quantify text descriptions and predict health ratings for hundreds of health states obtained through an online survey.

\section{Datasets and Overview of Variables}

\section{Health States and Their Online Text Explanations}

We first scraped online text explanations of health states from the National Health Service (NHS) website. NHS is the publicly funded healthcare system of the United Kingdom and the NHS website is one of the most popular online resources for medical advice in the United Kingdom. While there are a total of 1,175 headings listed under the "Health A-Z" section of NHS.uk, some of these are duplicate entries leading to the same informational web page with slightly different headings (e.g., "Aneurysm (Abdominal Aortic)" and "AAA," both lead to the same page). Two independent raters went through the list of all headings and excluded duplicates. These raters also excluded headings related to a procedure or a medication rather than an actual health state (e.g., "Abdominal Aortic Aneurysm Screening," "acupuncture," "aspirin”) as well as conditions that are only relevant to a particular population such as babies or children if a separate page exists for the more general condition in adults as well (e.g., "diabetes in pregnancy"). The inter-rater reliability for the exclusion decisions between the raters was very high, Cohen's Kappa $=0.93$ ( $p<.001)$. The raters resolved the remaining inconsistencies through a discussion.

After the exclusion process, we were left with 777 unique health states and their text explanations and discussions on the NHS website. These health states involve medical conditions and diseases of varying levels of severity, such as acne and brain aneurysm. In addition, each of the health states is associated with multiple sentences of summary information $(M=3.88, S D=$ 2.30 ) in the form of an explanation and/or a broad discussion, as well as many pages of additional details. Our analyses and tests use only the summary information, which is presented 
as the very first paragraph and is thus the first thing people who visit the website see. The summary information uses the least technical and most succinct language about the health state.

\section{Deaths and DALYs}

We obtained two different measures of objective health outcomes: mortality data and disability-adjusted life years (DALYs). These were taken from the Global Health Data Exchange website (http://ghdx.healthdata.org/gbd-results-tool), and we used statistics for all ages and genders from the year 2017. There were overlapping health states $(N=77$ with mortality data and $N=96$ with DALYs) that existed in both the Global Health Data Exchange website and the NHS website. These health states were used to evaluate the extent to which easily quantifiable, objective measures of health predict health perceptions.

\section{Frequency in Language}

We also measured the frequency with which the disease states are mentioned in everyday language. For this purpose, we queried the Google Books Ngram Dataset (version 2), one of the largest freely available resources of written natural language. This dataset includes word frequency statistics for billions of phrases (that are up to five words long) obtained through eight million books digitized by Google. We were able to obtain such statistics for 704 of 777 of our health states, which were present in the Google Books dataset. We log-transformed this frequency statistic before using it in our analyses.

\section{Text Features}

We measured 26 text features that have been previously shown to be important for the perception and interpretation of textual data, using the TextAnalyzer tool (Berger, Sherman, and Unger 2020). These features include the Flesch-Kincaid Grade Level (i.e., how many years of education it requires to understand; Kincaid et al. 1975), concreteness (i.e., how much a word refers to an actual, tangible, or "real” entity; Paetzold and Specia 2016), familiarity (i.e., how often a word is typically seen; Paetzold and Specia 2016), and emotionality (i.e., the degree to which an individual's attitude or reaction is based on emotion; Rocklage, Rucker, and Nordgren 2016). When given the health explanation text of a health state, the TextAnalyzer provides a score for each of these features. These scores are the weighted sums over the words in the text, with weights given by the relative frequencies of the words in a corresponding lexicon (see Humphreys and Wang 2018 and Berger et al. 2020 for details of this approach) 


\section{Embeddings of Health States}

Sentence Embeddings. We used a state-of-the-art language model called DistilBERT (Sanh et al. 2019) to represent our NHS text explanations as high-dimensional vectors. This model is specifically designed to extract multi-dimensional sentence vectors. It is a small, fast, cheap, and light Transformer model trained by distilling BERT, an earlier multi-layer bidirectional Transformer sentence encoder that has shown to be highly accurate at quantifying the meanings of sentences (Vaswani et al. 2017; Radford et al. 2018, 2019; Liu et al. 2019). BERT has two foundational steps in its framework: the pre-training and the fine-tuning. First, during the pre-training state, it is trained with two large corpora of passages on two tasks (a cloze task and a sentence ordering task). It uses masked language models with the goal of predicting the original ids of randomly masked tokens based on their context and predicting a sentence based on the previous sentence in a corpus. The pre-trained BERT model can then be fine-tuned with an additional output layer to successfully accommodate many different natural language processing tasks. DistilBERT gave us a 768-dimensional vector representation for each sentence in each text explanation. We averaged these sentence-level representations to have a single 768dimensional sentence vector for each health state.

Word Embeddings. We also used the Word2Vec model (Mikolov et al. 2013), an earlier language model that provides embedding representations for individual words (rather than sentences). Word2Vec applies a combination of the continuous bag-of-words and skip-gram algorithms, which ensure that words that appear in similar contexts and share related meanings are located close to each other in Word2Vec semantic space as well. This semantic space is derived from a corpus of a very large (100-billion-word) number of Google News articles. Previous work has demonstrated that word embeddings obtained through Word2Vec successfully predict human similarity judgment, associative judgment, and other related cognitive phenomena (see Turney and Pantel 2010; Young et al. 2018, Jones et al. 2015; Lenci 2018; Mandera, Keuleers, and Brysbaert 2017; Bhatia 2017a).

In order to obtain an embedding representation for each health state, we took the respective text explanation of a health state, tokenized all the sentences, and passed their words through Word2Vec to retrieve a high dimensional vector for each word. Then, we averaged these word-level representations to have a single 300-dimensional word embedding for each health state. 


\section{Health States Ratings Survey}

We can use the variables described in the preceding section as predictors in a regression model, with the outcome variable being lay participants' ratings of the severity of health states. While we have the necessary statistical and machine learning tools to construct such models based on embeddings obtained from text explanations and other language and health statistics, we needed to obtain human ratings of health states to train the model. We obtained these ratings using an online study.

\section{Methods}

Our study was run on Prolific Academic. In the study, participants $(N=782 \mathrm{UK}$ residents; 484 female, 291 male, 7 other; mean age $=35.12$ ) were asked to read NHS summaries for ten randomly selected health states (drawn from the pool of 777 health states). Then, they were asked to imagine that they were diagnosed with each of the health states and to report their evaluations of the health states by responding to the following prompt: "We would like to know how good or bad your health would be with [HEALTH STATE]. Using the 100-point scale below, please indicate how you think your health would be." These evaluations required them to slide a scale with labels "The worst health you can imagine" at point 0 and "The best health you can imagine" at point 100. Each health state summary and its associated rating question were present on a separate page, in a random order. Each health state was rated by an average of 10 participants $(S D=0.92)$ but the overall range for number of ratings varied from 6 to 12. In our analyses, we averaged all health state judgments for each health state across the participants.

Our experimental prompt (i.e., "We would like to know how good or bad your health would be with [HEALTH STATE]") is a variant of the standard EQ-5D's visual analogue scale (VAS). The EQ-5D is a well-established, commonly used, and trusted generic measure to gain a patient's global assessment of health and health-related quality of life in a wide variety of settings (Rabin, Oemar, and Oppe 2011; Feng, Parkin, and Devlin 2014). Our visual analogue scale (VAS) had a vertical, calibrated, line, anchored at 0 , the "worst health state imaginable" and at 100, the "best health state imaginable." This simple scale is commonly used for various health-related assessments. For example, one of its major uses is in health technology assessment; the UK's influential National Institute for Health and Clinical Excellence has declared the EQ-5D to be "the preferred measure of HRQL in adults." Due to its common use and simplicity, we believe that it is an appropriate measure of lay perceptions of health states. 


\section{General Statistical Methodology}

First, we built a regression model to see whether health-related measures such as the number of deaths, DALYs, language frequency, or other text features are good predictors of lay people's judgments of health. In particular, we used these measures as predictor variables in multiple regression models and computed each models' out-of-sample correlations using leaveone-out cross validation methods (details of cross validation provided below).

We also built a computational model to map the health states embeddings to lay people's judgments. Since the vector representations have 300 or more dimensions (based on the specific language model, DistilBERT or Word2Vec, used), we built a Ridge regression instead of a standard linear regression. A Ridge regression is a type of regularized regression that assumes a linear relationship between the predicted variable (mean lay people judgments of health states, $\mathrm{y}_{\mathrm{i}}$ ) and observable features (embeddings for health states, $\mathrm{X}_{\mathrm{i}}$ ), but penalizes the model fits based on sum-of-squares of the weight vector. This penalty is useful for avoiding any multicollinearity problems that are common with such high-dimensional data. In this project, we fit our Ridge regression using Python's scikit learn machine learning library (Pedregosa et al. 2011), and adopted all of scikit learn's default assumptions regarding the implementation of the Ridge regression (including setting $\alpha=1$ and allowing for a flexible additive intercept for predicting $\mathrm{yi})$.

In all of our predictive model evaluations, we used the leave-one-out cross validation method to test whether our approach of using health state embeddings generalizes well to out-ofsample data. Leave-one-out cross validation partitions the $N$ observations in the data into two portions: training data ( $N-1$ observations) and the test datum (the one remaining observation). The regression models are fit to the training data, and the resulting best-fit coefficients are used to predict the test datum from the model's predictor variables. This is repeated $N$ times so that each observation serves as the test datum once. We applied this procedure to the $N=777$ health states and quantified the predictive power of our models using the correlation and mean squared error between predicted and observed average lay people judgments of health states. Using a cross validation method is useful for two main reasons. First, since some of our predictor variables (such as health state embeddings) are very high dimensional, the resulting models may be overly flexible and not generalizable. Second, it is especially important for the future 
applications of our model to new health states or new health state explanations for which we have little or no survey data.

\section{Results}

\section{Overview of data}

The average lay people's judgments for each health state $\left(\mathrm{y}_{\mathrm{i}}\right)$ ranged from 1.82 to 90.89 , with a mean of 47.39 and a standard deviation of 19.97. Figure 1 shows the distribution of the mean lay people health ratings for all of the health states and the gray dashed line indicates the mean rating across all health states. On this figure, we also indicate the top five health states that are perceived to lead to "the worst health state imaginable" (or most severe) and "the best health state imaginable" (or least severe). Unsurprisingly, conditions such as brain hemorrhage or multiple system atrophy are rated by our participants to lead to the worst health states imaginable, while others such as black eyes or blisters are predicted to lead to the best health state imaginable in this context. In Figure 2, we visualize the 15 lowest (red downward pointing arrows) and highest rated (green upward pointing arrows) health states after applying multidimensional scaling to their sentence embeddings to recover latent dimensions that represent the health states. Looking at the graph, we observe that the health states cluster based on their perceived severity. In addition, health states that are semantically similar to each other (such as head and neck cancer, brain tumour, and brain abscess) are also close to one another. Thus, it is clear that our underlying vector space is accurately representing the intuitive similarity between health states as well as some variability in health state ratings.

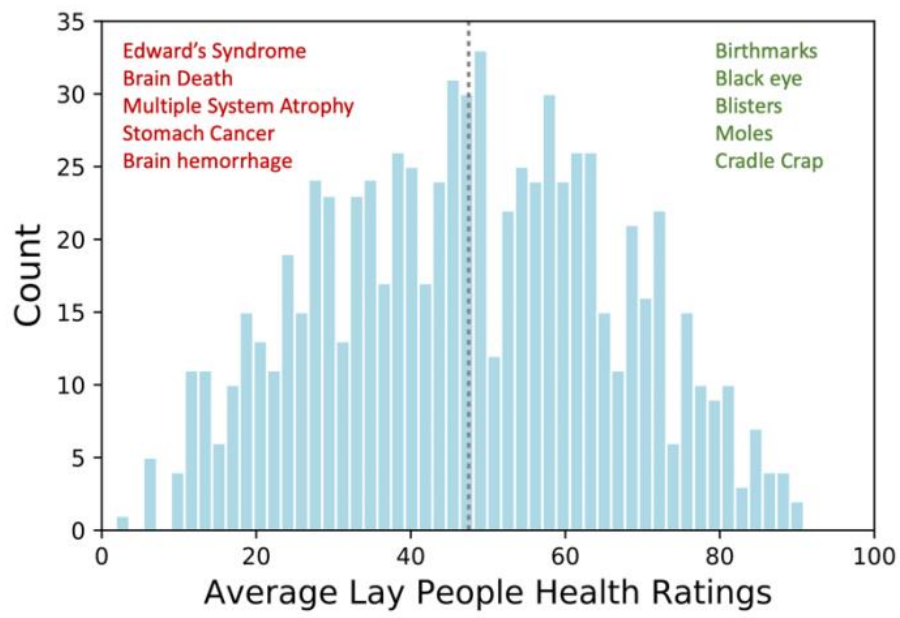

Figure 1. Histogram of the average lay people health ratings for all health states.

The top five health states that are perceived to lead to "the worst health state imaginable" (or 
most severe) and "the best health state imaginable" (or least severe) are indicated. In addition, the gray dashed line represents the mean health rating across all health states.

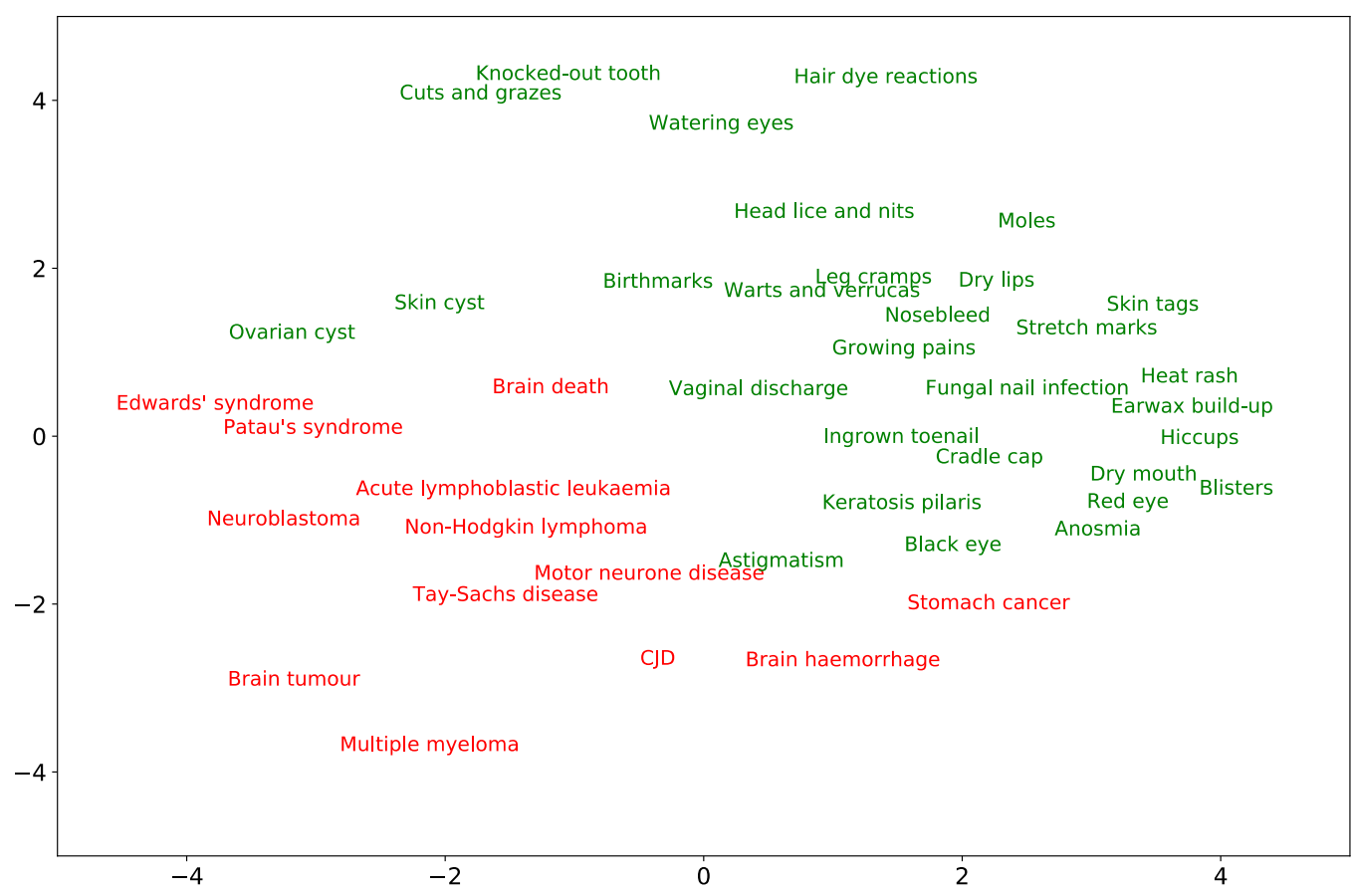

Figure 2. The underlying vector space appears to accurately represent the similarity between health states and capture some variability in health state ratings. Lowest and highest rated health states are visualized in the semantic space after applying multi-dimensional scaling to their (DistilBERT) embeddings. Health states are color-coded based on their average ratings (red health states are rated to be the most severe and green health states are rated to be the least severe).

\section{Performance of Predictor Variables}

First, we examined how the number of deaths, DALYs, and language frequency variables correlate with average perceptions of health. As expected, DALYs and health perceptions have a (marginally significant) negative relationship $(r=-0.189, p=0.065)$. Since one DALY can be thought of as one lost year of "healthy" life, it makes sense that health states with higher DALY scores are perceived to be more severe and receive lower ratings. Similarly, there is a negative relationship between the language frequency of a health state and ratings of the health state $(r=-$ $0.114, p=0.002)$. That is, health states that are more frequently used in language are judged to be more severe and thus receive lower ratings. There is no relationship between number of 
deaths caused by a health state and the ratings of the health state $(r=-0.060, p=0.602)$ in our sample. However, it is important to note that our analyses with the mortality data and DALYs only included $\sim 10 \%$ of the NHS health states (as most NHS health states were not present in other data sources).

Next, we investigated the out-of-sample predictive power of these variables using leaveone-out cross validation, with individual regressions for each of the three variables. These tests revealed an out-of-sample correlation of $r=-0.446(p<.001)$ for number of deaths, $r=0.095(p$ $=0.358)$ for DALYs, and $r=0.089(p=0.018)$ for language frequency (corresponding to RMSE $=14.332$ for number of deaths, RMSE $=16.279$ for DALYs, RMSE $=18.747$ for language frequency). Replicating prior work, these analyses demonstrate how objective measures may not be reliable predictors of human judgments of health severity. These results are shown in the top panels of Figure 3.

We also attempted an out-of-sample predictive analysis of the 26 text features (concreteness, sentiment etc.). Here we again used a leave-one-out cross validation with a multiple regression model. This model performed fairly well, obtaining an out-of-sample correlation of $r=0.550(p<.001$ and RMSE $=15.854)$ (see top right panel of Figure 3 ). This performance indicates that text explanations provide unique predictive power when studying perceptions of health states. With this in mind, we attempted a similar analysis using our DistilBERT sentence and Word2Vec word embedding models. Again, these models used a (Ridge) regression and attempted to predict the rating for a held out health state. In the bottom left panels of Figure 3, we plot the average participant judgments for each health state against embedding models' out-of-sample prediction for that health state along with those from alternative models. As visible in these scatterplots, our model predictions have a large out-ofsample correlation $(r=0.725, p<0.001$ for DistilBERT and $r=0.694, p<0.001$ for Word2Vec) and a smaller root-mean-squared-error $(\mathrm{RMSE}=13.245$ for DistilBERT and RMSE $=14.327$ for Word2Vec).

\section{Comparison with alternate metrics and models}

As shown in the previous section, the out-of-sample correlations obtained through our embedding models are very high, especially in comparison to the predictive power of models that use the other health-related and text measures. Here we evaluate these correlations by calculating three alternative metrics based directly on our participant data. The first metric we 
examined is mean pairwise participant correlation. This metric is computed by selecting pairs of participants and calculating the correlations between their health state judgments (across multiple pairs) to determine how well, on average, one participant's health state judgments predict another participant's health state judgments. To do so, we randomly sampled 1,000 pairs of participants from our dataset and measured the correlation for the health states that both participants in each pair rated. This analysis yielded a mean pairwise participant correlation of $r$ $=0.570(p<0.001)$, which is far less than our embedding models' accuracy rate (though slightly higher than that of our simple text features).

Second, we computed the expected participant-to-aggregate correlation. This metric measures the degree to which an individual participant's health state judgments are correlated with the aggregate health state judgments of all remaining $(\mathrm{M}-1)$ participants. We implemented this for all 782 participants and found an average participant-to-aggregate correlation of $r=$ 0.709 ( $p<0.001)$. This correlation is comparable to our Word2Vec model's predictive accuracy, yet still lower than our DistilBERT model's accuracy.

Finally, we measured the split-half reliability in our participant data, which quantifies the degree to which the aggregated judgments of half the participants are correlated with the other half of participants. To calculate the split-half reliability, we randomly divided the participants into two groups, calculated each group's average health state judgments, and finally calculated the correlation between these two sets of mean health state perceptions. As the split-half reliability metric determines how well half the participant sample predicts the health state judgments of the other half, it can be thought as a theoretical upper bound on the predictive accuracy attainable by any single model applied to the data in a very large sample of participants. Our procedure of randomly splitting the sample into two equal-sized groups 1,000 times yielded an average split-half reliability of $r=0.748$ ( $p<0.001)$, which is only slightly larger than the out-of-sample correlation achieved by our computational model using DistilBERT sentence embeddings.

In the bottom right bar graph of Figure 3, we plot our computational models' out-ofsample correlations against the correlations obtained through the alternative metrics, the pairwise participant, participant-to-aggregate, and split-half reliability correlations. Based on these results, we can conclude that our model's ability to predict the mean health state judgments of human participants is almost at the highest predictive power possible for these data. 

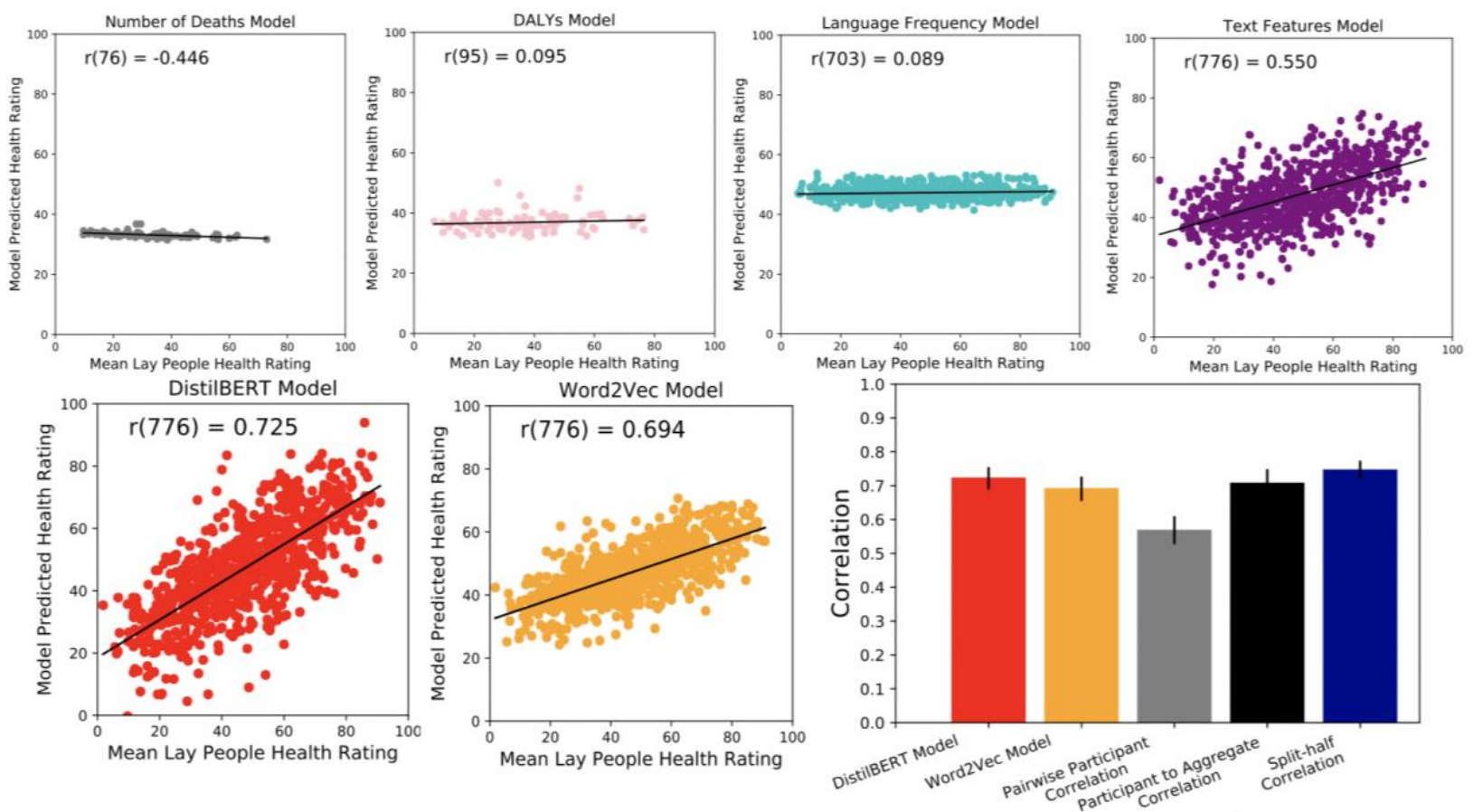

Figure 3. Leave-one-out cross validation-based correlations for each of our

predictive models, as well as various alternative metrics. Each scatter plot shows the mean participant judgments for each health state against our various model out-of-sample predictions for that health state. The dashed red lines shows the best linear fit of the data. The standard parametric correlation between the two variables $(r)$ is also presented. The bar plot on the bottom right demonstrates the predictive power achieved by the DistilBERT model (red bar), Word2Vec model (orange bar) and other comparison metrics, the pairwise participant (gray bar), participantto-aggregate (black bar), and split-half reliability (dark blue bar) correlations.

\section{Study 2: Understanding Health Judgments}

In the first part of our paper, we predicted health state judgments using word and sentence embeddings of online health explanations. Our models performed much better than various objective measures of health state severity (e.g. number of deaths, DALYs) and simple text features (e.g. concreteness, sentiment), and achieved close to the maximum predictive power possible in our dataset (i.e. by achieving correlations very close to the split-half correlation on participant ratings).

The natural next question in our investigation was to interpret the information contained in health state text explanations that gives rise to these successful predictions. Here we do this 
using our word embedding model. Recall that word embeddings are based on word cooccurrence statistics in natural language, and they quantify the extent to which words and concepts are associated with each other in language, and more generally, in the minds of people. We can thus use word embeddings to examine the types of words (in online text explanations) that most strongly predict high (and low) health state judgments. To make such an analysis manageable, we limit it to words in an established language dictionary, Linguistic Inquiry and Word Count (LIWC) lexicon (Pennebaker et al. 2001), health-relevant keywords and constructs compiled through an online study, the 500 most frequently used words from the NHS health state explanations, and a comprehensive list of body parts.

\section{Keywords and Psychological Constructs}

\section{Linguistic Inquiry and Word Count (LIWC)}

LIWC is an established language dictionary that is typically used to investigate the links between language and various psychological variables. LIWC has tens of different psychological categories and constructs, each consisting of hundreds of keywords. For example, the Social Processes category in LIWC includes keywords such as "mate" and "talk", while the Health construct includes keywords such as "clinic", "flu", and "pill". In traditional uses of the LIWC dictionary, the keywords in each construct are used in automated text analysis to quantify the degree to which these constructs and concepts are reflected in a given text (for a review, see Pennebaker, Mehl, and Niederhoffer 2003).

\section{Health-Related Keywords Survey}

The LIWC constructs are a good start for investigating the psychological dimensions underlying health judgment. Yet, it is necessary to add additional constructs and their keywords to confirm and build on top of the previous literature. Thus, in our next study, we asked participants to generate keywords that reflect various target constructs shown to be at play in health perception. Specifically, we recruited participants $(N=30$ UK residents; 19 female, 11 male; mean age $=30.53$ ) through Prolific Academic. In the study, participants read the following experimental prompt: "What are words that you would use to describe (or associate with) diseases with [CONSTRUCT]? Please write as many words as you can, separated with a comma in the form of [word1, word2, word3, ...]." The eight constructs we asked about in this study were those linked to perceptions of poor health in previous literature: death, functional inability, disability negative mood, anxiety, pain, physical distress, depression (Idler 1993; Kaplan and 
Camacho 1983; Fýlkesnes and Førde 2002; Farmer and Ferraro 1997; Garrity, Somes, and Marx 1978). Participants were paid at a rate equivalent to $\$ 9 /$ hour and did not receive any additional bonuses. Similarly, to the LIWC dictionary, this study yielded a set of keywords associated with various constructs, though the constructs used corresponded to health-relevant categories rather than general psychological categories.

\section{Most Common Health Explanation Words}

We created an additional word list with the most frequently used words from NHS health state explanations and discussions for our interpretive analyses. More specifically, we combined all words from all health state overviews scraped from NHS and identified the 500 that are most frequently used across all these texts.

\section{Body Parts}

Finally, we included a complete list of Body Parts (e.g. "brainstem" and "jaw") since previous literature has emphasized the importance of physical functioning, and many different organs and body parts also appeared in the most frequently used NHS words list.

\section{Computational Methods}

We used the above lists to measure the degree to which different words and constructs map onto perceptions of disease severity. In order to do this, we first trained our Word2Vec embeddings model on the full dataset of health states and ratings using Ridge regression. Then, for a given target word (e.g. "ache"), we obtained the word's 300-dimensional Word2Vec embedding. Finally, we passed this high-dimensional vector through our trained model to obtain a health state prediction for the word. Intuitively, this prediction can be seen as a predicted health judgment for a text explanation consisting only of the target word. Words that are given high predictions are associated with high health ratings and less severe health states, whereas words that are given low predictions are associated with low ratings and very severe health states. For constructs consisting of multiple keywords, we computed the average health judgment prediction for that construct by averaging all of the predictions for the keywords within that construct. 


\section{Results}

\section{LIWC}

Figure 4 presents the top and bottom ten LIWC psychological constructs and their health judgment predictions obtained through our Word2Vec embeddings model. Looking at these LIWC constructs and their predictions, we immediately see that Death (e.g., "coffin" and "kill") leads to the worst health states imaginable. Following that, other constructs such as Work (e.g., “job" and "majors"), Numbers ("minutes", "thousand") and Power ("superior") are also associated with bad health states. We suspect that these constructs all emphasize the importance of health states in a quantifiable manner, leading to low health judgment predictions. By contrast, better health states are associated with informal language, such as filler words (e.g., "you know"), nonfluencies (e.g., "hmm"), and even swear words. Unsurprisingly, the use of informal language may signal that a health state is less important and thus lead to higher judgments of health. Here, it is important to note that our interpretation of the LIWC analysis is rather speculative, as most LIWC categories are not informative or meaningful in the context of health judgment.

A.

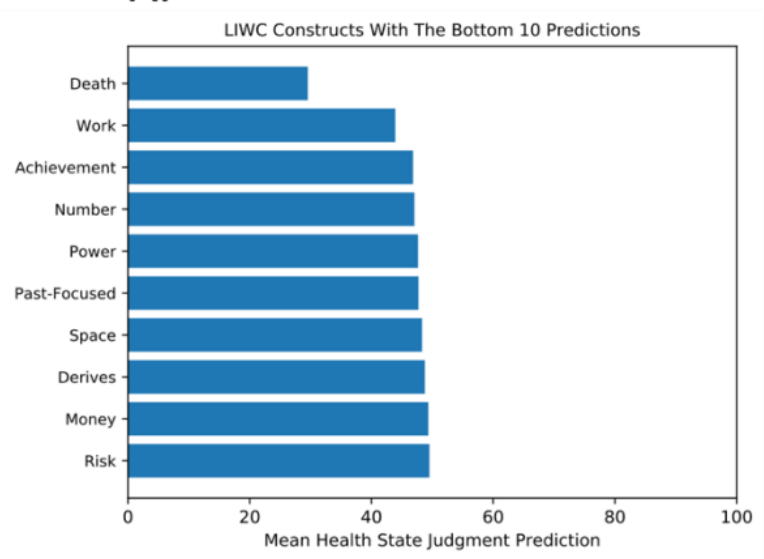

B.

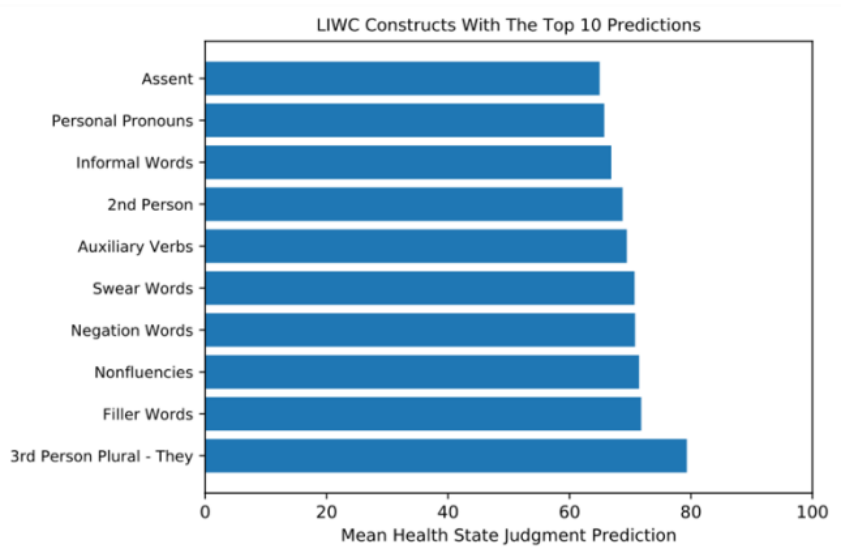

Figure 4. Bottom and Top Ten LIWC psychological constructs and their health judgment predictions obtained through our Word2Vec embeddings model. Sorted from predicted to lead to "the worst health state imaginable" (or the most severe) to "the best health state imaginable" (or the least severe). 


\section{Health-Related Keywords Survey}

Mean predictions for each construct using our computational model along with example participant generated keywords are shown in Figure 5. Confirming research findings from previous literature, we observed that death-related words received the lowest health judgments $($ mean prediction $=27.147)$, followed by functional inability $($ mean prediction $=28.845)$ and disability-related words (mean prediction $=33.838)$. However, pain $($ mean prediction $=43.970)$, physical distress $($ mean prediction $=44.687)$, and depression-related words $($ mean prediction $=$ 44.884) were perceived to be relatively less severe. While this may appear surprising at first glance, functional inability and disabilities that are long term poor conditions of health and may be judged to be very severe and disruptive to daily functioning. In contrast, pain may often be perceived as a temporary, ephemeral state that can be reduced with appropriate medical attention. Indeed, Goldstein, Siegel, and Boyer (1984) have found that acute conditions do not seem to have the expected negative impact on health judgment. Our model's predictions are consistent with this finding.

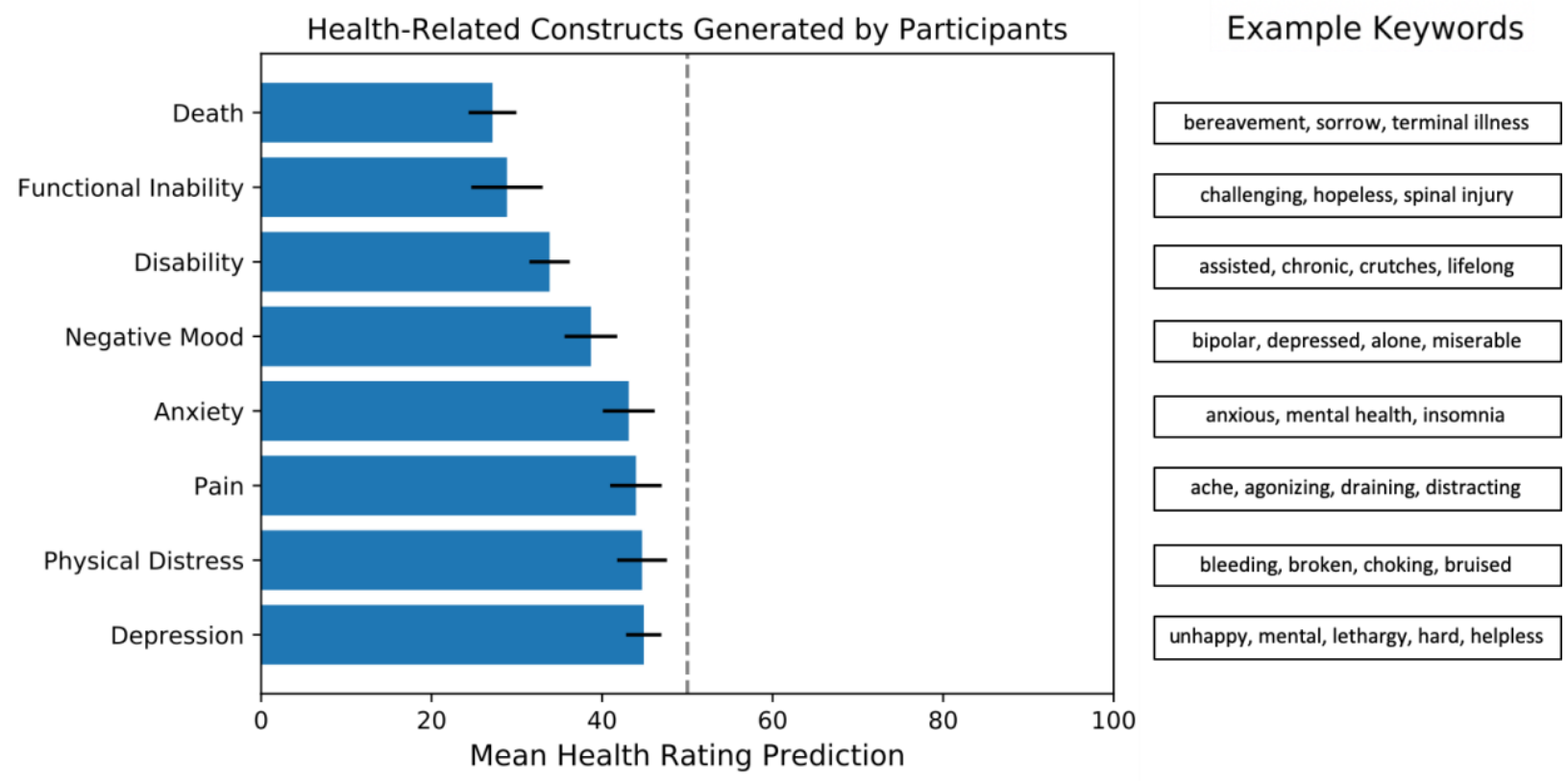

Figure 5. Health-related constructs participants and their mean health rating predictions obtained through our Word2Vec embeddings model. Example keywords from these constructs are presented in the right panel. 


\section{Most Common Health Explanation Words}

The word clouds in Figure 6 show individual words from the 500 most frequently used words in the online text overviews of health states that received the highest and lowest predictions by our Word2Vec embeddings model. Here we see that words signaling some uncertainty and less definitive, prescriptive language such as "occasionally," "tend," or "sometimes" lead to higher health state judgments. By contrast organ names such as "lung" and "heart", as well as other death related keywords such as "died" and "fatality" are more associated with low health judgments.

A.

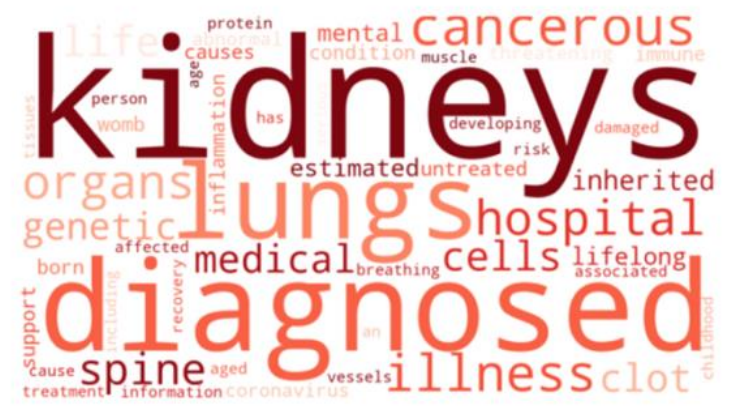

B.

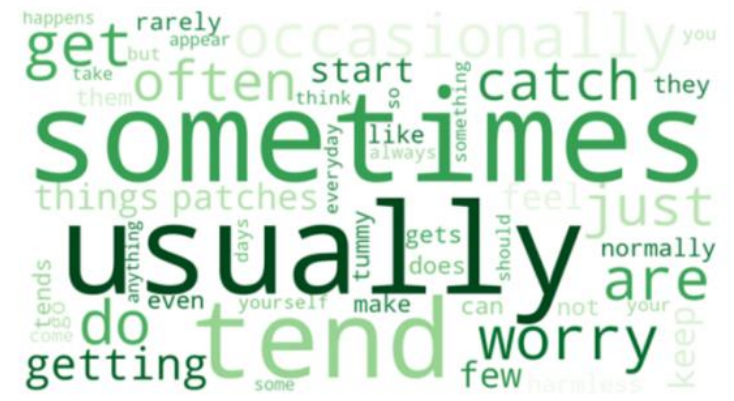

Figure 6. Frequently used words that received the lowest (red) and highest (green) predictions through our Word2Vec embeddings model.

\section{Body Parts}

In Figure 7, we present word clouds with the 50 body parts that lead to lowest and highest health judgment predictions obtained through our Word2Vec embeddings model. As seen from these word clouds, "brain," "liver," "bone marrow," and "kidney" are associated with bad health states while other body parts such as "eyelashes," "thumb," "lip," and "pinky" are associated with less severe health states.

Our findings highlighting the importance of different body parts are in parallel to Mavaddat et al.'s (2011) study which strongly associates physical functioning with self-rated health, more so than mental health and social functioning. Similarly, Simon et al. (2005) also showed that $80 \%$ of participants referred to one or more physical aspects when assessing their general health. These findings are in line with multiple studies demonstrating the close association with physical function measures and health outcomes (Sofi et al. 2008). 
A.

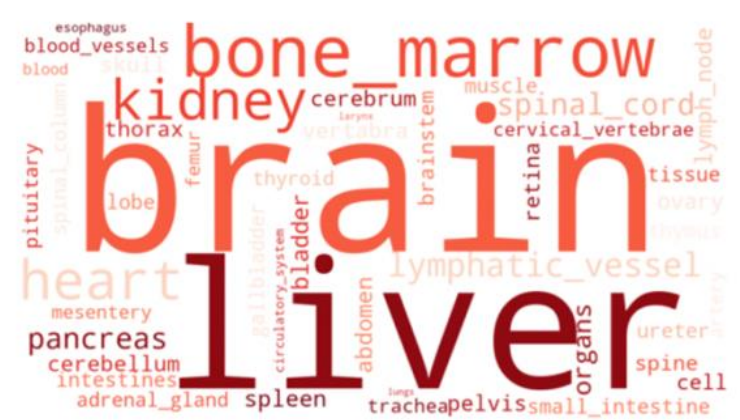

B.

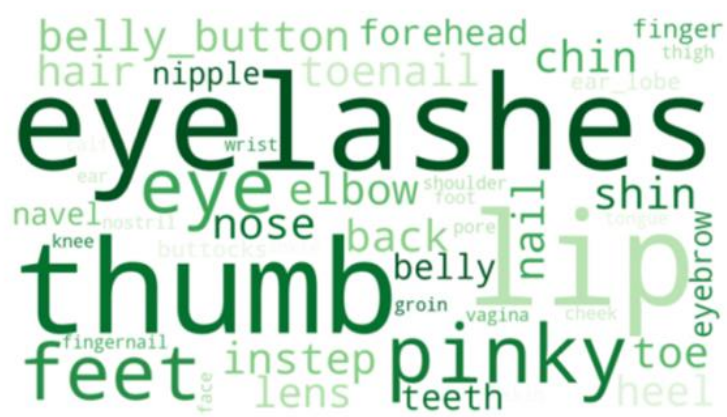

Figure 7. Body parts with the lowest (red) and highest (green) predictions through our Word2Vec embeddings model.

\section{Study 3: Predicting Differences between Health Explanations}

The above studies have shown the utility of our approach for predicting and interpreting health state judgments. Here we examine an application of this approach to health communication, focusing specifically on predicting differences in participant judgments for different health explanations of the same health state. Consider, for example, breast cancer. Unsurprisingly, there are explanations and discussions of breast cancer in many different online health forums, including both the NHS and the American Breast Cancer Foundation (abcf.org) websites. However, these two websites describe breast cancer differently, and it is possible that these different explanations lead to different judgments about the severity of breast cancer. If our approach is successful at describing health judgment, then it should be able to predict, in an a priori manner, the differences in judgments evoked by these two explanations. Such an a priori prediction, if successful, can have many practical applications in health risk communication and perception.

\section{Methods}

We identified six health states that have explanations on both NHS.uk as well as on other health-related websites. These health states include more severe conditions such as ankylosing spondylitis neuroma as well as less severe conditions such as bad breadth, and the health-related websites belonging to their respective organizations (i.e., spondylitis.org and ww.mouthhealthy.org). We extracted these explanations from each of the six health states. These explanations were offered to participants in an online study run on Prolific Academic. 
Participants $(N=80$ UK residents; 56 female, 24 male, 0 other; mean age $=36.16)$ were shown one of the six health states from the alternative health-related websites at random and were asked rate its severity using the same rating scale as in Study 1. We compared the health ratings for these six health states from the alternative websites in this study to those obtained through NHS.uk in Study 1. Here, it is important to mention that we matched the number of sentences in these overviews as closely as possible between NHS.uk and the alternative websites. A complete list of these overviews can be found in the Web Appendix.

\section{Results}

For each health state explanation on each website, we obtained model predictions from our DistilBERT sentence embeddings model that was trained on full data from Study 1 and an average health rating from our participants. Figure 8 demonstrates these predictions and the corresponding human ratings. As can be seen in this figure, different health state explanations led to different health perceptions, and thus average participant ratings varied depending on the explanation. Importantly, our embeddings model was able to successfully predict these differences. For all of the health states, the difference predicted by our model (the difference between red and green bars on the left side of the subplots) was also observed in the average human participant ratings (the difference between red and green bars on the right side of the subplots). These results show that our approach is able to predict, a priori, differences in perceptions of the health state as a function of the health state's explanation.
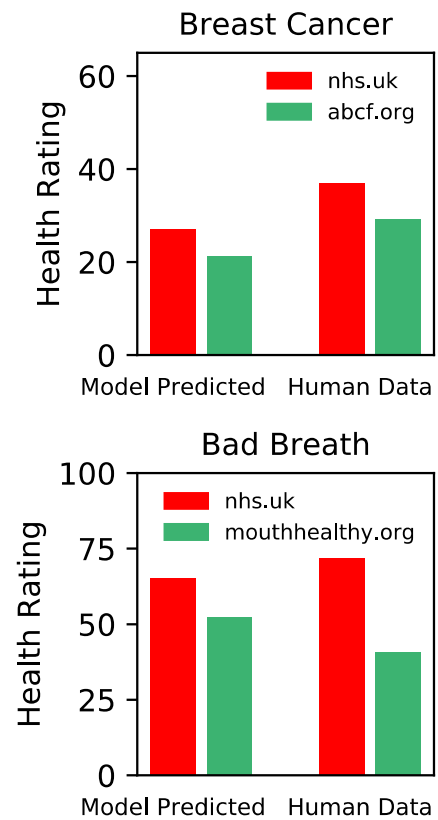

Acoustic Neuronoma

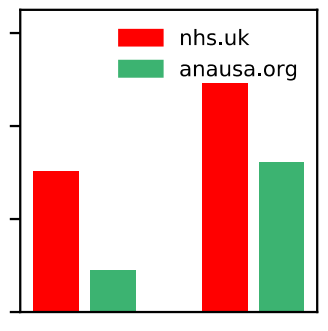

Model Predicted Human Data

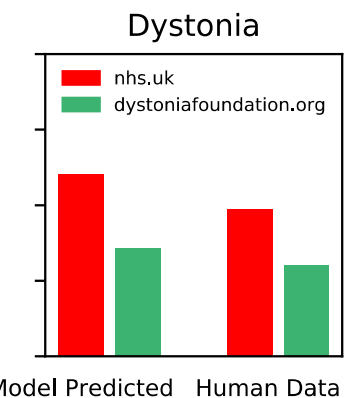

Ankylosing Spondylitis

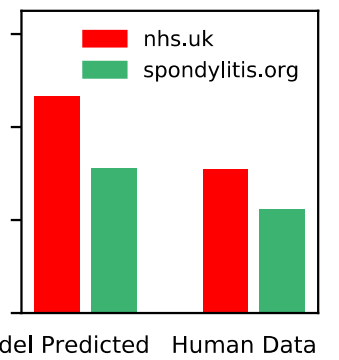

Model Predicted Human Data

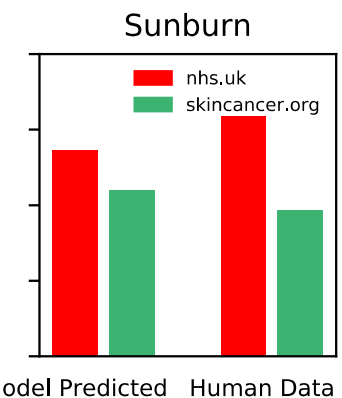


Figure 8. DistilBERT model predictions and average participant ratings for each of the organizational websites introducing the six different health states demonstrate how our embeddings model was able successfully predict these differences. For all of the health states, the difference observed in model predictions for the two websites (the difference between red and green bars on the left side of the subplots) was also observed in average human participant ratings (the difference between red and green bars on the right side of the subplots).

\section{DISCUSSION}

In this paper, we present a novel machine learning approach that aims to predict lay people's health judgments. Using recent advances in machine learning, specifically sentence and word embedding models, we quantified the information contained in online text overviews of hundreds of different health states found on the National Health Service (NHS) website. In Study 1, we mapped these sentence and word embeddings onto lay health perceptions collected through an online study. Our machine learning approach was able to accurately predict how participants perceived different health states. Using the DistilBERT and Word2Vec embeddings, our models achieved very high out-of-sample correlations between the predicted and observed health ratings. These accuracy rates were higher than those obtained from other predictors, such as number of deaths and DALYs, language frequency, and various simple text features, and they were also close to the split-half reliability correlation, which is the theoretical upper-bound in making such predictions. In Study 2, we used our best-fit models to analyze the language present in these text overviews and understand the determinants of health state judgments. For example, our model replicated previous empirical findings about the importance of constructs such as disability and death for health perception and extends them by discussing their respective contributions and how different body parts also relate to high and low health ratings. Finally, in Study 3, we demonstrated how our embeddings approach can be used to predict differences in health judgments associated with different explanations of a given health state.

Since self-assessed health is a complex, multidimensional concept (Simon et al., 2005), quantifying and understanding health judgment has been a challenging task for many years. In this paper, we showcased a powerful new technique that can easily be adopted by researchers, policymakers, and medical providers to predict health perceptions. In addition to successfully predicting health judgment, our method also offers many new applications and avenues for future 
research. For example, our approach can be used to study most optimal health communication formats as well as predictions of judgment errors. From a methodological perspective, adopting cutting-edge technologies from data science and creating opportunities to further integrate these techniques with consumer health behavior research is essential for scalable health prediction. Thus, we believe that our approach is a beneficial first step for projects with important consumer behavior implications and for improving the well-being of people.

It is important to mention that our study also has some limitations. First, despite the fact that the media and internet have a substantial influence on how people acquire information and make decisions, these are not the only sources people can rely on. In fact, while we focused our analysis on NHS.uk health overviews, most people are exposed to a wide variety of information sources, including discussions with medical providers, family members, and friends. Future work can try to obtain linguistic communication datasets involving these sources, and apply our embeddings approach to model individual differences in health behavior and subsequently quantify the effect of various different information sources on health judgment. Studying individual differences would also pave the way for targeted health communication and behavioral phenotyping. Second, our current approach does not analyze social network data, which is an important source for online health information (Gottlieb and Green 1984; CohenCole and Fletcher 2008; Eichstaedt et al. 2015; Surian et al. 2016). In future work, our methods can be applied to such data (e.g., messages on Twitter, Reddit and Facebook), and be used in conjunction with social network analysis to better predict lay health perception. Third, we did not take into account any demographic or socioeconomic variables while explaining the variance in health judgment. However, previous studies demonstrated that some of these variables such as employment status, education level, or retirement status may be important to consider while others such as gender may not be as much (Wan 1976; Undén and Elofsson 2006). While we did not collect demographic variables other than age and gender from our Prolific Academic participants, our methods can easily be applied to study differences in participant demographic and socioeconomic variables if the training data is collected from a target population.

To conclude, we present a novel, generalizable, and high performing method to predict and understand health judgment. More specifically, we mapped embeddings from online text explanations and discussions of different health states to laypeople's perceptions of health. Our method combines insights from data science and health behavior research and opens up many 
potential research questions with substantial real-world applications. We hope that researchers, policymakers, and medical professionals will use this approach to investigate many other exciting research questions related to judgments of health. 


\section{References}

Arnesen, Trude and Lydia Kapiriri (2004a), "Can the Value Choices in DALYs Influence Global Priority-Setting?," Health policy, 70(2), 137-49.

- (2004b), "Can the Value Choices in DALYs Influence Global Priority-Setting?," Health policy, 70(2), 137-49.

Atkinson, Nancy, Sandra Saperstein, and John Pleis (2009), "Using the Internet for Health-Related Activities: Findings from a National Probability Sample," Journal of medical Internet research, 11(1), e5.

Becker, Marshall H., Lois A. Maiman, John P. Kirscht, Don P. Haefner, and Robert H. Drachman (1977a), "The Health Belief Model and Prediction of Dietary Compliance: A Field Experiment," Journal of Health and Social behavior, 348-66.

_ (1977b), "The Health Belief Model and Prediction of Dietary Compliance: A Field Experiment," Journal of Health and Social behavior, 348-66.

Berger, Jonah, Ashlee Humphreys, Stephan Ludwig, Wendy W. Moe, Oded Netzer, and David A. Schweidel (2020), "Uniting the Tribes: Using Text for Marketing Insight," Journal of Marketing, $84(1), 1-25$.

Berger, Jonah, Garrick Sherman, and Lyle Ungar (2020), TextAnalyzer. Retrieved from http://textanalyzer.org.

Bhatia, Sudeep (2017), “Associative Judgment and Vector Space Semantics.," Psychological Review, 124(1), 1.

(2019), "Predicting Risk Perception: New Insights from Data Science," Management Science, 65(8), 3800-3823.

Brewer, Noel T., Gretchen B. Chapman, Frederick X. Gibbons, Meg Gerrard, Kevin D. McCaul, and Neil D. Weinstein (2007a), "Meta-Analysis of the Relationship between Risk Perception and Health Behavior: The Example of Vaccination.," Health psychology, 26(2), 136.

- (2007b), "Meta-Analysis of the Relationship between Risk Perception and Health Behavior: The Example of Vaccination.," Health psychology, 26(2), 136.

Calvert, Melanie J. and N. Freemantle (2003a), "Use of Health-related Quality of Life in Prescribing Research. Part 1: Why Evaluate Health-related Quality of Life?," Journal of clinical pharmacy and therapeutics, 28(6), 513-21.

_ (2003b), "Use of Health-related Quality of Life in Prescribing Research. Part 1: Why Evaluate Health-related Quality of Life?," Journal of clinical pharmacy and therapeutics, 28(6), $513-21$.

Chapman, Gretchen B. and Elliot J. Coups (2006a), "Emotions and Preventive Health Behavior: Worry, Regret, and Influenza Vaccination.," Health psychology, 25(1), 82.

— (2006b), "Emotions and Preventive Health Behavior: Worry, Regret, and Influenza Vaccination.," Health psychology, 25(1), 82.

Chapman, Gretchen B. and Arthur S. Elstein (2000a), "Cognitive Processes and Biases in Medical Decision Making," Decision making in health care: Theory, psychology, and applications, 183210.

(2000b), "Cognitive Processes and Biases in Medical Decision Making," Decision making in health care: Theory, psychology, and applications, 183-210.

Cheung, Helier (2020), "Coronavirus: Why Attitudes to Masks Have Changed around the World," BBC News, https://www.bbc.com/news/world-53394525. 
Cohen-Cole, Ethan and Jason M. Fletcher (2008), "Is Obesity Contagious? Social Networks vs. Environmental Factors in the Obesity Epidemic," Journal of health economics, 27(5), 1382-87. Corbett, Julia B. and Motomi Mori (1999a), "Medicine, Media, and Celebrities: News Coverage of Breast Cancer, 1960-1995," Journalism \& Mass Communication Quarterly, 76(2), 229-49. - (1999b), "Medicine, Media, and Celebrities: News Coverage of Breast Cancer, 1960-1995," Journalism \& Mass Communication Quarterly, 76(2), 229-49.

Cornwall, Andrea and Rachel Jewkes (1995a), "What Is Participatory Research?," Social science \& medicine, 41(12), 1667-76.

- (1995b), "What Is Participatory Research?," Social science \& medicine, 41(12), 1667-76.

Covello, Vincent T. and Richard G. Peters (2002), "Women's Perceptions of the Risks of AgeRelated Diseases, Including Breast Cancer: Reports from a 3-Year Research Study," Health Communication, 14(3), 377-95.

Devlin, Jacob, Ming-Wei Chang, Kenton Lee, and Kristina Toutanova (2018), "Bert: Pre-Training of Deep Bidirectional Transformers for Language Understanding," arXiv preprint arXiv:1810.04805.

Eichstaedt, Johannes C., Hansen Andrew Schwartz, Margaret L. Kern, Gregory Park, Darwin R. Labarthe, Raina M. Merchant, Sneha Jha, Megha Agrawal, Lukasz A. Dziurzynski, and Maarten Sap (2015), "Psychological Language on Twitter Predicts County-Level Heart Disease Mortality," Psychological science, 26(2), 159-69.

Entwistle, Vikki A., Mary J. Renfrew, Steven Yearley, John Forrester, and Tara Lamont (1998a), "Lay Perspectives: Advantages for Health Research," Bmj, 316(7129), 463-66. (1998b), "Lay Perspectives: Advantages for Health Research," Bmj, 316(7129), 463-66.

Farmer, Melissa M. and Kenneth F. Ferraro (1997), "Distress and Perceived Health: Mechanisms of Health Decline," Journal of Health and Social Behavior, 298-311.

Feng, Yan, David Parkin, and Nancy J. Devlin (2014), "Assessing the Performance of the EQ-VAS in the NHS PROMs Programme," Quality of Life Research, 23(3), 977-89.

Fýlkesnes, Knut and Olav Helge Førde (1992), "Determinants and Dimensions Involved in SelfEvaluation of Health," Social science \& medicine, 35(3), 271-79.

Garrity, Thomas F., Grant W. Somes, and Martin B. Marx (1978), "Factors Influencing SelfAssessment of Health," Social Science \& Medicine. Part A: Medical Psychology \& Medical Sociology, 12, 77-81.

Goldstein, Michael S., Judith M. Siegel, and Richard Boyer (1984), "Predicting Changes in Perceived Health Status.," American Journal of Public Health, 74(6), 611-14.

Gottlieb, Nell H. and Lawrence W. Green (1984), "Life Events, Social Network, Life-Style, and Health: An Analysis of the 1979 National Survey of Personal Health Practices and Consequences," Health Education Quarterly, 11(1), 91-105.

Gualtieri, Lisa N. (2009), "Improving Patient-Physician Communication about Internet Use: Why 'Don't Ask, Don't Tell' Doesn't Work," in Medicine 2.0 Conference, JMIR Publications Inc., Toronto, Canada.

Gualtieri, Lisa Neal (2009), "The Doctor as the Second Opinion and the Internet as the First," in CHI'09 Extended Abstracts on Human Factors in Computing Systems, 2489-98.

Günther, Fritz, Luca Rinaldi, and Marco Marelli (2019), "Vector-Space Models of Semantic Representation from a Cognitive Perspective: A Discussion of Common Misconceptions," Perspectives on Psychological Science, 14(6), 1006-33.

Harrison, Joel A., Patricia D. Mullen, and Lawrence W. Green (1992a), “A Meta-Analysis of Studies of the Health Belief Model with Adults," Health education research, 7(1), 107-16. 
(1992b), "A Meta-Analysis of Studies of the Health Belief Model with Adults," Health education research, 7(1), 107-16.

Hudacek, David L., Shyama Kuruvilla, Nora Kim, Katherine Semrau, Donald Thea, Shamim Qazi, Andrew Pleasant, and James Shanahan (2011a), "Analyzing Media Coverage of the Global Fund Diseases Compared with Lower Funded Diseases (Childhood Pneumonia, Diarrhea and Measles)," PLoS One, 6(6), e20438.

(2011b), "Analyzing Media Coverage of the Global Fund Diseases Compared with Lower Funded Diseases (Childhood Pneumonia, Diarrhea and Measles)," PLoS One, 6(6), e20438.

Humphreys, Ashlee and Rebecca Jen-Hui Wang (2018), “Automated Text Analysis for Consumer Research," Journal of Consumer Research, 44(6), 1274-1306.

Idler, Ellen L. (1993), "Perceptions of Pain and Perceptions of Health," Motivation and Emotion, 17(3), 205-24.

Idler, Ellen L., Shawna V. Hudson, and Howard Leventhal (1999), "The Meanings of Self-Ratings of Health: A Qualitative and Quantitative Approach," Research on aging, 21(3), 458-76.

Janz, Nancy K. and Marshall H. Becker (1984), "The Health Belief Model: A Decade Later," Health education quarterly, 11(1), 1-47.

Jones, Michael N., Jon Willits, Simon Dennis, and Michael Jones (2015), "Models of Semantic Memory," Oxford handbook of mathematical and computational psychology, 232-54.

Kaplan, George A. and Terry Camacho (1983), "Perceived Health and Mortality: A Nine-Year Follow-up of the Human Population Laboratory Cohort," American journal of epidemiology, 117(3), 292-304.

Katapodi, Maria C., Kathy A. Lee, Noreen C. Facione, and Marylin J. Dodd (2004a), "Predictors of Perceived Breast Cancer Risk and the Relation between Perceived Risk and Breast Cancer Screening: A Meta-Analytic Review," Preventive medicine, 38(4), 388-402.

- (2004b), "Predictors of Perceived Breast Cancer Risk and the Relation between Perceived Risk and Breast Cancer Screening: A Meta-Analytic Review," Preventive medicine, 38(4), 388402.

Keller, Punam Anand and Donald R. Lehmann (2008), "Designing Effective Health Communications: A Meta-Analysis," Journal of Public Policy \& Marketing, 27(2), 117-30.

Kincaid, J. P., R. P. Fishburne, R. L. Rogers, and B. S. Chissom (1975), "Flesch-Kincaid Grade Level," Memphis: United States Navy.

Krause, Neal M. and Gina M. Jay (1994), "What Do Global Self-Rated Health Items Measure?," Medical care, 930-42.

Lenci, Alessandro (2018), "Distributional Models of Word Meaning," Annual review of Linguistics, 4, 151-71.

Liu, Xiaodong, Pengcheng He, Weizhu Chen, and Jianfeng Gao (2019), “Multi-Task Deep Neural Networks for Natural Language Understanding," arXiv preprint arXiv:1901.11504.

Mandera, Paweł, Emmanuel Keuleers, and Marc Brysbaert (2017), "Explaining Human Performance in Psycholinguistic Tasks with Models of Semantic Similarity Based on Prediction and Counting: A Review and Empirical Validation," Journal of Memory and Language, 92, 57-78. Mikolov, Tomas, Ilya Sutskever, Kai Chen, Greg S. Corrado, and Jeff Dean (2013), "Distributed Representations of Words and Phrases and Their Compositionality," Advances in neural information processing systems, 26, 3111-19.

Organization, World Health (1999a), The World Health Report: 1999: Making a Difference, World Health Organization. (1999b), The World Health Report: 1999: Making a Difference, World Health Organization. 
Paetzold, Gustavo and Lucia Specia (2016), "Inferring Psycholinguistic Properties of Words," in Proceedings of the 2016 Conference of the North American Chapter of the Association for Computational Linguistics: Human Language Technologies, 435-40.

Pennebaker, James W., Martha E. Francis, and Roger J. Booth (2001), "Linguistic Inquiry and Word Count: LIWC 2001," Mahway: Lawrence Erlbaum Associates, 71(2001), 2001.

Pennebaker, James W., Matthias R. Mehl, and Kate G. Niederhoffer (2003), "Psychological Aspects of Natural Language Use: Our Words, Our Selves," Annual review of psychology, 54(1), 547-77.

Peters, Ellen and Louise Meilleur (2016a), "The Influence of Affect on Health Decisions," in Handbook of Health Decision Science, Springer, 109-20. (2016b), "The Influence of Affect on Health Decisions," in Handbook of Health Decision Science, Springer, 109-20.

Powell, John, Nadia Inglis, Jennifer Ronnie, and Shirley Large (2011), "The Characteristics and Motivations of Online Health Information Seekers: Cross-Sectional Survey and Qualitative Interview Study," Journal of medical Internet research, 13(1), e20.

Rabin, Rosalind, Mandy Oemar, Mark Oppe, Bas Janssen, and Michael Herdman (2011), "EQ-5D-5L User Guide," Basic information on how to use the EQ-5D-5L instrument. Rotterdam: EuroQol Group, 22.

Radford, Alec, Karthik Narasimhan, Tim Salimans, and Ilya Sutskever (2018), "Improving Language Understanding with Unsupervised Learning," Technical report, OpenAI.

Radford, Alec, Jeffrey Wu, Rewon Child, David Luan, Dario Amodei, and Ilya Sutskever (2019), "Language Models Are Unsupervised Multitask Learners," OpenAI blog, 1(8), 9.

Reyna, Valerie F. (2020a), "A Scientific Theory of Gist Communication and Misinformation Resistance, with Implications for Health, Education, and Policy," Proceedings of the National Academy of Sciences.

- (2020b), "Of Viruses, Vaccines, and Variability: Qualitative Meaning Matters," Trends in Cognitive Sciences.

Rocklage, Matthew D., Derek D. Rucker, and Loran F. Nordgren (2018), "Persuasion, Emotion, and Language: The Intent to Persuade Transforms Language via Emotionality," Psychological Science, 29(5), 749-60.

Rogers, Anna, Olga Kovaleva, and Anna Rumshisky (2020), “A Primer in Bertology: What We Know about How Bert Works," arXiv preprint arXiv:2002.12327.

Rothman, Alexander J. and Peter Salovey (1997), "Shaping Perceptions to Motivate Healthy Behavior: The Role of Message Framing.," Psychological bulletin, 121(1), 3.

Sanh, Victor, Lysandre Debut, Julien Chaumond, and Thomas Wolf (2019), "DistilBERT, a Distilled Version of BERT: Smaller, Faster, Cheaper and Lighter," arXiv preprint arXiv:1910.01108.

Sheeran, Paschal, Peter R. Harris, and Tracy Epton (2014a), "Does Heightening Risk Appraisals Change People's Intentions and Behavior? A Meta-Analysis of Experimental Studies.," Psychological bulletin, 140(2), 511.

- (2014b), "Does Heightening Risk Appraisals Change People's Intentions and Behavior? A Meta-Analysis of Experimental Studies.," Psychological bulletin, 140(2), 511.

Simon, Jeanette G., J. B. De Boer, I. M. A. Joung, Hans Bosma, and J. P. Mackenbach (2005), "How Is Your Health in General? A Qualitative Study on Self-Assessed Health," The European Journal of Public Health, 15(2), 200-208.

Slovic, Paul, Melissa Finucane, Ellen Peters, and Donald G. MacGregor (2002a), "Rational Actors or Rational Fools: Implications of the Affect Heuristic for Behavioral Economics," The Journal of Socio-Economics, 31(4), 329-42. 
(2002b), "Rational Actors or Rational Fools: Implications of the Affect Heuristic for

Behavioral Economics," The Journal of Socio-Economics, 31(4), 329-42.

Slovic, Paul and Ellen Peters (2006a), "Risk Perception and Affect," Current directions in psychological science, 15(6), 322-25.

(2006b), "Risk Perception and Affect," Current directions in psychological science, 15(6), $322-25$.

Sofi, Francesco, Andrea Capalbo, Francesca Cesari, Rosanna Abbate, and Gian Franco Gensini (2008), "Physical Activity during Leisure Time and Primary Prevention of Coronary Heart Disease: An Updated Meta-Analysis of Cohort Studies," European Journal of Cardiovascular Prevention \& Rehabilitation, 15(3), 247-57.

Surian, Didi, Dat Quoc Nguyen, Georgina Kennedy, Mark Johnson, Enrico Coiera, and Adam G. Dunn (2016), "Characterizing Twitter Discussions about HPV Vaccines Using Topic Modeling and Community Detection," Journal of medical Internet research, 18(8), e232.

Turney, Peter D. and Patrick Pantel (2010), "From Frequency to Meaning: Vector Space Models of Semantics," Journal of artificial intelligence research, 37, 141-88.

Undén, Anna-Lena and Stig Elofsson (2006), "Do Different Factors Explain Self-Rated Health in Men and Women?," Gender medicine, 3(4), 295-308.

Vahabi, Mandana (2007), "The Impact of Health Communication on Health-related Decision Making," Health Education.

Van Bavel, Jay J., Katherine Baicker, Paulo S. Boggio, Valerio Capraro, Aleksandra Cichocka, Mina Cikara, Molly J. Crockett, Alia J. Crum, Karen M. Douglas, and James N. Druckman (2020a), "Using Social and Behavioural Science to Support COVID-19 Pandemic Response," Nature Human Behaviour, 1-12.

- (2020b), "Using Social and Behavioural Science to Support COVID-19 Pandemic Response," Nature Human Behaviour, 1-12.

Van Der Pligt, Joop (1998a), "Perceived Risk and Vulnerability as Predictors of Precautionary Behaviour," British journal of health psychology, 3(1), 1-14.

- (1998b), "Perceived Risk and Vulnerability as Predictors of Precautionary Behaviour," British journal of health psychology, 3(1), 1-14.

Vaswani, Ashish, Noam Shazeer, Niki Parmar, Jakob Uszkoreit, Llion Jones, Aidan N. Gomez, Łukasz Kaiser, and Illia Polosukhin (2017), "Attention Is All You Need," in Advances in Neural Information Processing Systems, 5998-6008.

Wan, Thomas T. (1976), "Predicting Self-Assessed Health Status: A Multivariate Approach.," Health Services Research, 11(4), 464.

Weinstein, Neil D. (2000a), "Perceived Probability, Perceived Severity, and Health-Protective Behavior.," Health Psychology, 19(1), 65.

- (2000b), "Perceived Probability, Perceived Severity, and Health-Protective Behavior.," Health Psychology, 19(1), 65.

Young, Tom, Devamanyu Hazarika, Soujanya Poria, and Erik Cambria (2018), "Recent Trends in Deep Learning Based Natural Language Processing," ieee Computational intelligenCe magazine, 13(3), 55-75. 